\title{
Primary extraskeletal mesenchymal chondrosarcoma of the vulva
}

\author{
Eun Ae Jeh ${ }^{1}$, Young Jin Lee ${ }^{1}$, Heung Yeol Kim ${ }^{1}$, Ari Kim², Jun Hee Lee ${ }^{2}$ \\ ${ }^{1}$ Department of Obstetrics and Gynecology, Kosin University College of Medicine, Busan; ${ }^{2}$ Institute of Wonkwang Medical Science, Wonkwang \\ University College of Medicine, Iksan, Korea
}

Extraskeletal chondrosarcoma is rare, making up only $1 \%$ of reported chondrosarcoma. We experienced 3 cases of extraskeletal chondrosarcoma, especially in vulva. They were suspected as lipoma of the vulva. The patients had noticed a small but growing mass on their vulva which had been palpated earlier. The masses were excised with a $2 \mathrm{~cm}$ resection margin. The final pathological reports confirmed extraskeletal mesenchymal chondrosarcoma (EMC) of the vulva revealing no microscopic lesions on the resection margins. After 24 months of following from the initial diagnosis, the patients remain without evidence of any recurrent. Management of EMC is not well studied due to the rare and variable nature of the disease. However, the surgery, such as we had, is the mainstay of local treatment with studies showing better survival in patients who undergo wide surgical resection. The establishment of adjuvant systemic pharmacotherapy could be expected in the future.

Keywords: Chondrosarcoma; Extraskeletal; Vulva

\section{Introduction}

Extraskeletal chondrosarcoma which is rare, accounting for only $1 \%$ to $3 \%$ of all vulvar malignancies, has been considered as primary sarcomas of the vulva. The most common vulvar sarcomas are leiomyosarcomas, rhabdomyosarcomas, angiosarcomas, neurofibrosarcomas, malignant fibrohistiocytomas, and aggressive angiomyxomas [1]. Among primary vulvar sarcomas, primary extraskeletal mesenchymal chondrosarcomas (EMC) are extremely rare, and have higher rate of misdiagnosis. There is little information about the behavior and optimal management strategy for EMC of the vulva. To the best of our knowledge, there have only been two cases (1 Japanese and 1 African American) of primary EMC of the vulva reported in English literature [2,3].

In the present study, we describe three cases of very rare EMC of the vulva in a Korean postmenopausal woman, with the expectation of better strategy for the diagnosis and treatment.

\section{Case reports}

\section{Case 1}

A 68-year-old woman was referred to our clinic with a di- agnosis of a mass of the vulva. The patient had noticed a small but growing mass on her right vulva which had been palpated one month earlier. On physical examination, a globular, movable mass measuring about $4 \mathrm{~cm}$ in diameter was palpated in the right major labium. The mass was excised with a $2 \mathrm{~cm}$ resection margin. Frozen section biopsy of the

Received: 2013.4.5. Revised: 2013.5.13. Accepted: 2013.6.10. Corresponding authors: Jun Hee Lee

Department of Obstetrics and Gynecology, Wonkwang University Sanbon Hospital, Wonkwang University School of Medicine, 327 Sanbon-ro, Gunpo 435-040, Korea

Tel: +82-31-390-2360 Fax: +82-31-390-2244

E-mail: ttochil@hanmail.net

Heung Yeol Kim

Department of Obstetrics and Gynecology, Kosin University Gospel Hospital, Kosin University College of Medicine, 262

Gamcheon-ro, Seo-gu, Busan 602-702, Korea

Tel: +82-51-990-3300 Fax: +82-51-990-3300

E-mail: hykyale@yahoo.com

Articles published in Obstet Gynecol Sci are open-access, distributed under the terms of the Creative Commons Attribution Non-Commercial License (http://creativecommons. org/licenses/by-nc/3.0/) which permits unrestricted non-commercial use, distribution, and reproduction in any medium, provided the original work is properly cited.

Copyright $\odot 2013$ Korean Society of Obstetrics and Gynecology 


\section{Obstetrics \& Gynecology Science}

Vol. 56, No. 5, 2013
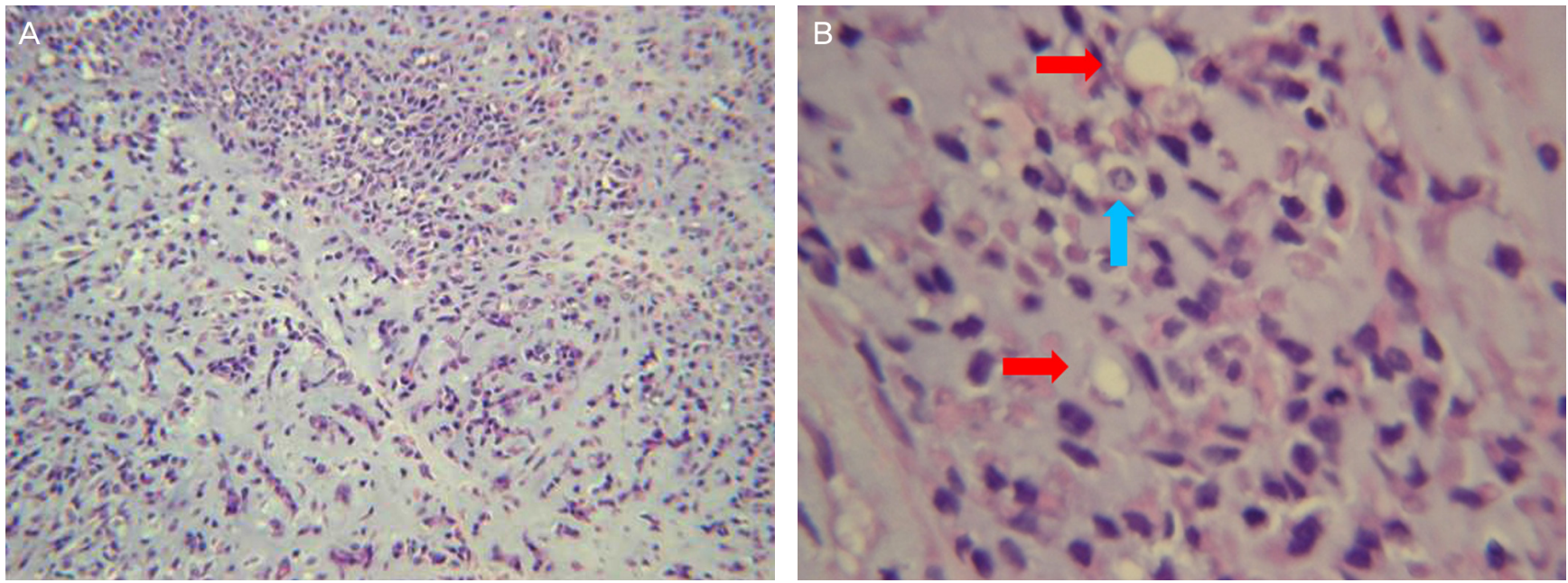

Fig. 1. (A) Hematoxylin and eosin stained photomicrograph of undifferentiated tumor cells with areas of chondroid differentiation ( $\times 25)$. (B) Hematoxylin and eosin stained photomicrograph showing islands of mature hyaline cartilage (red arrows) mixed with undifferentiated, small, round, mesenchymal cells (blue arrow) $(\times 40)$.

resected specimen demonstrated a chondrosarcoma with a negative resection margin. The final pathological report confirmed a $6 \times 2.8 \times 2.3 \mathrm{~cm}$ sized EMC of the vulva revealing no microscopic lesions on the resection margins (Fig. 1). Tumor markers were within normal range (carcinoembryonic antigen [CEA], CA-19-9, CA-125, and alpha fetoprotein [AFP]). Immunohistochemistry was performed on the tumor to analyze the expression of vimentin, S-100 protein, desmin, and epithelial membrane antigen (EMA). The tumor was strongly positive for vimentin and S-100 protein but negative for the other markers. The patient recovered from surgery without any postoperative complications. Bone scans and chest radiographs for disease staging were negative. Thirty months following from the initial diagnosis, the patient revealed no evidence of recurrence as assessed by pelvic magnetic resonance imaging (MRI) and chest radiographs.

\section{Case 2}

A 52-year-old, postmenopausal woman visited our clinic with a complaint of a mass of the vulva. She had noticed a small but growing mass on her left vulva which had been palpated 2 months earlier. On physical examination, a globular, movable mass measuring about $2 \mathrm{~cm}$ in diameter was palpated in the left major labium. The mass was excised with a $2 \mathrm{~cm}$ resection margin. The pathological report confirmed a $4 \times 3.8 \times 2$ $\mathrm{cm}$ sized EMC of the vulva revealing no microscopic lesions on the resection margins. Immunohistochemistry was performed on the tumor to analyze the expression of vimentin, S-100 protein, desmin, Pan-CK, smooth muscle actin, and EMA. She recovered from surgery without any complications. Bone scans and chest radiographs for disease staging were negative. After twenty four-month of following the initial diagnosis, the patient remains without evidence of any recurrent disease as assessed by pelvic MRI and chest radiographs.

\section{Case 3}

A 59-year-old woman was referred to gynecologic clinic with a diagnosis of the lipoma of the left vulva. She identified a small but growing mass before one month. A globular, movable mass measuring about $3 \mathrm{~cm}$ in diameter was palpated in the left major labium. The mass was excised with a $2 \mathrm{~cm}$ resection margin. Frozen section biopsy of the resected specimen demonstrated a chondrosarcoma with a negative resection margin. The final pathological report confirmed a $4 \times 4 \times 3$ $\mathrm{cm}$ sized EMC of the vulva revealing no microscopic lesions on the resection margins. Tumor markers were within normal range (CEA, CA-19-9, CA-125, and AFP). Immunohistochemistry was performed on the tumor to analyze the expression of vimentin, S-100 protein, desmin, and EMA. The tumor was strongly positive for vimentin, but negative for the other markers. The patient recovered from surgery without any postoperative complications. Bone scans and chest radiographs for disease staging were negative. After twenty four-month of following-up, she did not present any recurrence as assessed by pelvic MRI and chest radiographs. 


\section{Obstetrics \& Gynecology Science}

Eun Ae Jeh, et al. Extraskeletal mesenchymal chondrosarcoma of the vulva

\section{Discussion}

Sarcomas of the vulva are extremely rare and account for only $1 \%$ to $3 \%$ of all vulvar malignancies [3]. It is a slow growing malignant tumor, and the lesions apparent in vulvar sarcomas are frequently misdiagnosed as Bartholin's cysts or abscesses and the median age at diagnosis is around fifty years. The most common presenting symptom is local discomfort due to a vulvar mass, chronic vulvar pruritus, or both and patients can also present with pain associated with intercourse $[4,5]$. The histopathologic characteristics are multi-nodular growth of primitive chondroid cells and the abundant myxoid matrix [4].

While surgery remains the mainstay of treatment for vulvar sarcomas, radical vulvectomy carries a high risk of short and long term morbidity [6]. However, a limited wide local excision may result in a positive or close margin requiring the use of adjuvant therapy. Prognosis seems to depend on the size of the lesion, tumor involvement of adjacent tissue, and mitotic activity. Women with lesions greater than $5 \mathrm{~cm}$ in diameter, with infiltrating margins, extensive necrosis and with more than five mitotic figures per 10 high power fields, are more likely to have recurrent disease following surgical excision $[1,7]$.

Mesenchymal chondrosarcoma is a rare, but highly aggressive variant of chondrosarcoma. It has a poor prognosis with high rate of local recurrence and protracted lifetime with distant metastasis [8]. Compared to conventional chondrosarcomas which are very rarely extraskeletal and occur later in life, mesenchymal chondrosarcomas frequently have an extraskeletal location and present at an earlier age [4].

Histologically, EMC display a biphasic pattern comprising sheets of undifferentiated, round or spindle mesenchymal cells, interspersed with islands of hyaline cartilage. The differential diagnosis for EMC includes synovial sarcoma, EWing sarcoma, hemangiopericytoma and a dedifferentiated chondrosarcoma, particularly based on biopsy specimens. The identification of cartilage in the biopsy helps in differentiating EMC from the first three differentials listed and an intermixing of the cartilaginous elements with undifferentiated small round cell components helps in differentiating EMC from a dedifferentiated chondrosarcoma [9].

Immunohistochemistry also plays a vital role in diagnosing EMC. Immunopositivity for vimentin, S-100 and Mic-2 (CD 99) and negativity for cytokeratin, EMA and CD 34 is characteris- tic for EMC [10].

The tendency of EMC is to locally recur with occasional distant metastasis to the lung. According to Antonescu et al. [11], in their reports of twenty patients with extraskeletal chondrosacoma, $75 \%$ of patients had local recurrence or metastatic recurrence and the most common metastatic site was lung. Also they described that EMC are aggressive, resulting in $35 \%$ to $50 \%$ mortality within 5 years and an average disease free interval of 20 months. Disease has been reported to recur up to 8 years out from diagnosis. Thus, the patients with EMC need adequate or extended excision and long term surveillance with pelvic examination and with pelvic MRI and chest radiographs to evaluate recurrence. Furthermore, the efficacy of radiotherapy and chemotherapy is presently considered debatable. EMC have yet to display a consistent response to any kinds of systemic chemotherapy used for other soft tissue sarcoma.

Management of EMC is not well studied due to the rare and variable nature of the disease, but treatment plans are evolving and several strategies have been identified throughout the literature. The current management consensus underscores the importance of both local and systemic control. Surgery, such as we had, is the mainstay of local treatment with studies showing better survival in patients who undergo wide surgical resection $[8,12]$.

In our case, the patients exhibited classic clinical presentation along with a characteristic bimorphic histological appearance and the immunohistochemical findings helped in confirming the diagnosis of EMC. The responses for the special staining were different among them. They were treated only with wide local excision, without recurrence for at least twenty four months. Some suggested that the prognosis of EMC is defined by distant metastasis developing more than 10 years later, but not by local recurrence [13].

In the prior two reports of a gynecological case of EMC, the first patient was treated by surgery with a wide local excision, similar to our patient, and the second patient underwent radical treatment. Both these patients reported to be without evidence of recurrent disease for 6 months and 2 years respectively.

With respect to our patients, we performed wide local wide excision with a $2 \mathrm{~cm}$ negative margin for treatment. Postoperative radiotherapy or chemotherapy was not thought to be necessary because the efficacy of radiotherapy and chemotherapy is presently considered debatable [3]. Twenty-four 


\title{
Obstetrics \& Gynecology Science
}

\author{
Vol. 56, No. 5, 2013
}

months following the initial diagnosis, the patients remain without evidence of any recurrent disease as assessed by pelvic MRI and chest radiographs. To our best of knowledge, this is the first report that reports over 2 years' follow up after only wide excision.

Based on this case and previous reports [2,3], we suggest that local wide excision could be enough for the treatment of chondrosarcoma of vulva. However, to check the metastatic recurrence, long-term follow-up is needed. Thus, the establishment of adjuvant systemic pharmacotherapy could be expected according to the cases.

\section{Conflict of interest}

No potential conflict of interest relevant to this article was reported.

\section{Acknowledgments}

This study was supported by Wonkwang University in 2012.

\section{References}

1. Nirenberg A, Ostor AG, Slavin J, Riley CB, Rome RM. Primary vulvar sarcomas. Int I Gynecol Pathol 1995;14:5562.

2. Santacruz MR, Proctor L, Thomas DB, Gehrig PA. Extraskeletal myxoid chondrosarcoma: a report of a gynecologic case. Gynecol Oncol 2005;98:498-501.

3. Sawada M, Tochigi N, Sasajima Y, Hasegawa T, Kasamatsu T, Kitawaki J. Primary extraskeletal myxoid chondrosarcoma of the vulva. J Obstet Gynaecol Res
2011;37:1706-10.

4. Ulutin HC, Zellars RC, Frassica D. Soft tissue sarcoma of the vulva: a clinical study. Int J Gynecol Cancer 2003;13:528-31.

5. Patnayak R, Manjulatha B, Srinivas S, Reddy MK, Sambasivaiah $\mathrm{K}$, Jena $\mathrm{A}$. Leiomyosarcoma of the vulva. Indian J Pathol Microbiol 2008;51:448-9.

6. Magne N, Pacaut C, Auberdiac P, Jacquin JP, Chargari C, Chauleur $C$, et al. Sarcoma of vulva, vagina and ovary. Best Pract Res Clin Obstet Gynaecol 2011;25:797-801.

7. Allen J. The clinical nurse specialist in gynaecological oncology-the role in vulval cancer. Best Pract Res Clin Obstet Gynaecol 2003;17:591-607.

8. Shakked RJ, Geller DS, Gorlick R, Dorfman HD. Mesenchymal chondrosarcoma: clinicopathologic study of 20 cases. Arch Pathol Lab Med 2012;136:61-75.

9. Shapeero LG, Vanel D, Couanet D, Contesso G, Ackerman LV. Extraskeletal mesenchymal chondrosarcoma. Radiology 1993;186:819-26.

10. Jain A, Safaya R, Jagan C, Sharma SK. Extraskeletal mesenchymal chondrosarcoma of the pleura: report of a rare case. Indian J Pathol Microbiol 2011;54:144-6.

11. Antonescu CR, Argani P, Erlandson RA, Healey JH, Ladanyi M, Huvos AG. Skeletal and extraskeletal myxoid chondrosarcoma: a comparative clinicopathologic, ultrastructural, and molecular study. Cancer 1998;83:1504-21.

12. Cesari M, Bertoni F, Bacchini P, Mercuri M, Palmerini E, Ferrari S. Mesenchymal chondrosarcoma: an analysis of patients treated at a single institution. Tumori 2007;93:423-7.

13. Kawaguchi S, Wada T, Nagoya S, Ikeda T, Isu K, Yamashiro K, et al. Extraskeletal myxoid chondrosarcoma: a multi-institutional study of 42 cases in Japan. Cancer 2003;97:1285-92. 